\title{
Primary hepatic tuberculosis: A rare occurrence
}

\author{
Devayani Niyogi, Mahesh Goel, Rajesh S Shinde, and Shraddha Patkar
}

\author{
GI \& HPB Service, Department of Surgical Oncology, Tata Memorial Hospital, Mumbai, India
}

\begin{abstract}
Primary hepatic tuberculosis is a rare entity and can closely mimic malignancy with respect to clinical presentation and imaging features. We identified five patients at a high volume tertiary care cancer center, whose clinical features and imaging closely mimicked primary liver malignancy or metastases but final histopathology was suggestive of hepatic tuberculosis. Three patients underwent a surgical resection whereas two were diagnosed on a biopsy. Anti-tuberculosis therapy was started for all the patients which was well tolerated. All patients are doing well at the time of the last follow up. This case series stresses the importance of having a high index of suspicion and preoperative biopsy in cases where imaging features are equivocal. (Ann Hepatobiliary Pancreat Surg 2019;23:80-83)
\end{abstract}

Key Words: Primary hepatic tuberculosis; Mimic; Malignancy

\section{INTRODUCTION}

Tuberculosis (TB) affects the liver either as a part of widespread miliary tuberculosis or, it can be primary hepatic tuberculosis, i.e. affliction of the liver by the TB bacillus (Mycobacterium tuberculosis) in the absence of pulmonary or miliary TB. ${ }^{1}$ This clinical entity is extremely rare even in countries endemic for tuberculosis. Primary hepatic tuberculosis can closely mimic malignancy with respect to clinical presentation and imaging features. ${ }^{2}$ It is essential to keep a high index of suspicion, especially in endemic regions, to avoid unnecessary surgery and start prompt treatment in the form of anti-tubercular therapy (ATT).

\section{CASE}

\section{Patients}

From our multidisciplinary liver clinic records in a dedicated hepatobiliary oncology unit we identified five patients, whose clinical features and imaging closely mimicked primary liver malignancy or metastases but final histopathology was suggestive of hepatic TB.

\section{Case 1}

A 43-year-old gentleman presented with a lump in left lumbar and hypochondriac region associated with pain and no history of fever. Contrast-enhanced computerized tomography (CECT) scan showed a mass arising from the left kidney with a lesion in segment VI of the liver and no evidence of disease elsewhere. The liver lesion appeared to be metastatic on radiology review. Clinico-radiologically diagnosis of oligometastatic renal cell carcinoma (RCC) was considered, and as the lesion was resectable, no tissue diagnosis was attempted.

The patient underwent a left radical nephrectomy with a synchronous resection of the hepatic segment VI lesions guided by intraoperative ultrasound (IOUS). The final histopathology report showed papillary RCC with the liver lesion reported as necrotizing granulomatous inflammation. Stain for acid-fast bacilli (AFB) was negative. The patient was given 4-drug ATT. He is doing well and is on regular follow-up.

\section{Case 2}

A 53-year-old lady with diabetes mellitus, a known case of RCC of the right kidney had undergone a partial

Received: June 13, 2018; Revised: October 14, 2018; Accepted: October 22, 2018

Corresponding author: Shraddha Patkar

GI \& HPB Services, Department of Surgical Oncology, Tata Memorial Hospital, Homi Bhabha National Institute, Ernest Borges Marg, Parel, Mumbai 400 012, India

Tel: +91-22-2417 7172, Fax: +91-22-2414 8114, E-mail: drshraddhapatkar@gmail.com

Copyright (C) 2019 by The Korean Association of Hepato-Biliary-Pancreatic Surgery

This is an Open Access article distributed under the terms of the Creative Commons Attribution Non-Commercial License (http://creativecommons.org/ licenses/by-nc/4.0) which permits unrestricted non-commercial use, distribution, and reproduction in any medium, provided the original work is properly cited. Annals of Hepato-Biliary-Pancreatic Surgery • pISSN: 2508-5778 • elSSN: 2508-5859 
nephrectomy in August 2014. On routine follow up, ultrasound showed a lesion in the right lobe of the liver. CECT showed a lesion in the sub-capsular region of segment VII of the liver. Positron emission tomography-comuted tomography showed low-grade uptake (standardized uptake value [SUV] 6.71) in the liver lesion (Fig. 1). Fine needle aspiration cytology (FNAC) from the liver lesion was inconclusive. She underwent wide excision of the liver lesion. Final histopathology report was suggestive of necrotizing granulomatous lesions of probable tubercular etiology. Chest computed tomography (CT) did not show any lesions that could signify the primary site of TB. She was started on 4-drug ATT. She is healthy and on follow-up.

\section{Case 3}

A 59-year-old gentleman with diabetes mellitus presented to us with abdominal pain and weight loss. On evaluation, CECT scan and magnetic resonance imaging (MRI) the of abdomen showed a lesion occupying the entire left lobe of the liver $(11 \times 7 \mathrm{~cm})$ with features suggestive of intra-hepatic cholangiocarcinoma (Fig. 2). FNAC of the lesion done elsewhere was suggestive of adenocarcinoma. He underwent a left hepatectomy. IOUS did not show any satellite lesion. Final histopathology report showed necrotizing granulomatous inflammation with Langhans type giant cells (Fig. 3). Stain for AFB was negative. He too had no history or pulmonary focus of TB. He was started on hepatosafe ATT (2 SHLE/7 HLE

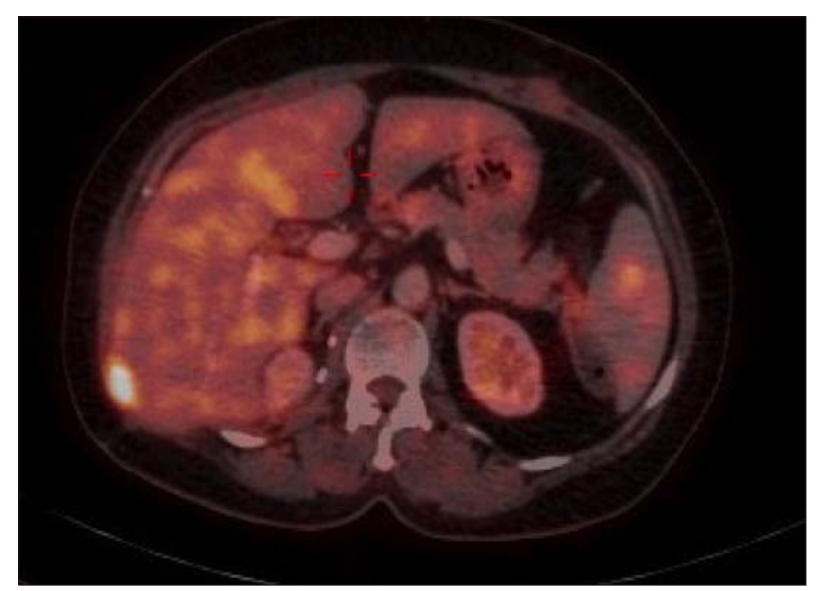

Fig. 1. Positron-emission tomography-computed tomography image of case 2, showing intense fluorodeoxyglucose uptake in the liver lesion. daily regimen) in view of post-hepatectomy status. He is doing well and on follow up.

\section{Case 4}

A 61-year-old hypertensive lady presented to our clinic with a history of fever, loss of weight and appetite and generalized malaise. Screening ultrasonography of the abdomen showed multiple lesions in the liver suspicious for metastases. A CECT abdomen and pelvis showed suspicious nodularity in the cecum in addition to the liver lesions. FNAC from the liver lesion done before referral was suspicious for malignancy. Colonoscopy revealed no pathology in the cecum. A biopsy from the liver lesion showed non-necrotizing epithelioid granulomas. Special

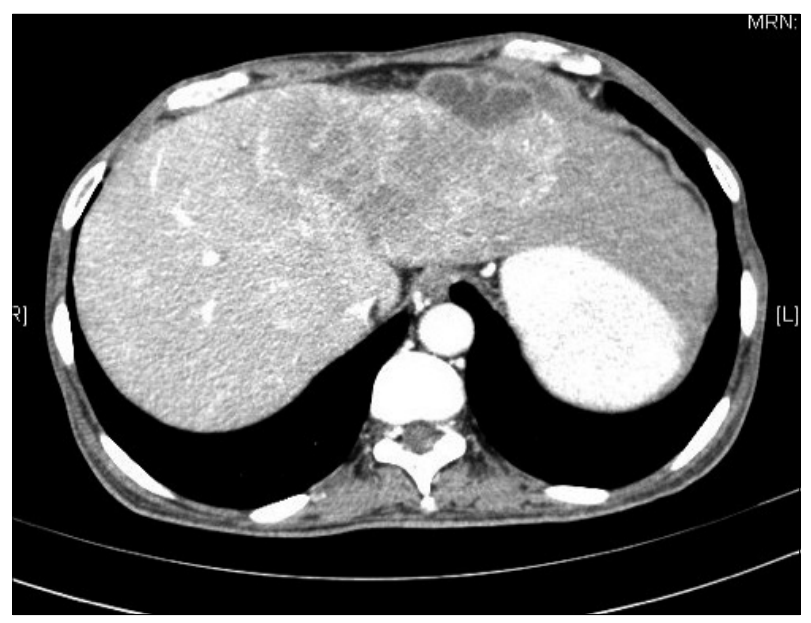

Fig. 2. Contrast-enhanced computed tomography image of case 3, showing enhancing lesion in left lobe of the liver.

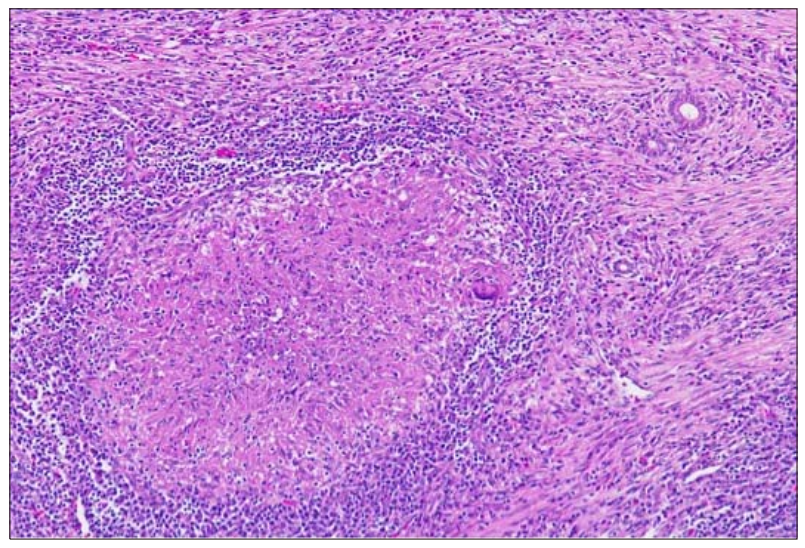

Fig. 3. Typical morphological features of tubercular granuloma on electron microscopy showing Langhans giant cell, necrosis and a biliary radicle (H\&E, 10×). 
stain for AFB was negative. She was started on ATT and is currently doing well.

\section{Case 5}

A 48-year-old gentleman presented with a history of pain in the abdomen, fever, night sweats and weight loss. A CECT scan showed a solitary, poorly enhancing lesion in the caudate lobe of liver with few peri-portal nodes. MRI confirmed a lesion in caudate lobe $(6.9 \times 6.4 \times 5.8 \mathrm{~cm})$ hyperintense on $\mathrm{T} 2 \mathrm{~W}$ and minimally hyperintense on $\mathrm{T} 1 \mathrm{~W}$ images with restricted diffusion. A peripherally enhancing capsule with multiple necrotic areas without any arterial or porto-venous enhancement was seen.

Tumour markers and liver function tests were standard. Given atypical imaging, a biopsy was performed which revealed necrotizing granulomatous inflammation. Special stains for AFB were negative. He was started on 4-drug ATT following which appetite and weight improved, constitutional symptoms resolved and is currently doing well on follow-up.

\section{DISCUSSION}

Hepatic involvement in tuberculosis has been classified by Levine as follows: Hepatic involvement in military TB; hepatic involvement in pulmonary TB; primary hepatic TB; focal tuberculoma or abscess; and tubercular cholangitis.

Hepatic involvement in military TB is the most common form occurring in $50-80 \%$ of cases due to the dissemination of the TB bacilli through the hepatic artery. ${ }^{3}$

Primary hepatic TB is a rare entity. ${ }^{1-5}$ Ours is a tertiary cancer center with a high volume of liver resections (approximately 100 resections per year). However, we have come across only five such cases of primary hepatic TB. Such a small number in an endemic country like India highlights the rare nature of this clinical entity.

The proposed mechanism for primary hepatic TB is that it begins as primary intestinal TB with a small ulcer in the small bowel. This ulcer acts as an access point for the TB bacilli to gain entry into the portal vein and seed the liver parenchyma. The primary ulcer then undergoes complete resolution, manifesting as primary hepatic TB. However, the low oxygen tension in the liver does not make it a favorable environment for the TB bacillus to thrive. ${ }^{4}$ The rich blood supply and the presence of the reticuloendothelial system in the liver, facilitate granuloma formation once seeding of the TB bacillus occurs.

Most of these granulomas are periportal in location. They cause minimal interference to normal hepatic function, therefore, most of these lesions are asymptomatic. The cases reported in literature mostly presented with diffuse epigastric pain, anorexia, weight loss or low-grade fever. $^{2,4-7}$ Some were asymptomatic. ${ }^{2}$ In the 5 cases reported by us, 2, which were suspected to be metastases from renal cell carcinoma were asymptomatic, the third patient presented with abdominal pain and weight loss whereas the other two patients presented with classic constitutional symptoms of tuberculosis.

There are no characteristic imaging features for hepatic tubercular lesions. They can mimic primary liver malignancy or metastases. ${ }^{2,3}$ Although a biopsy is not mandated for diagnosis of hepatocellular carcinoma; imaging needs to be unequivocal.

Any atypical features should prompt tissue the diagnosis. In our series, the first two cases appeared to be metastases from the renal origin on imaging. These patients underwent small non-anatomical liver resections. The third patient appeared classically like intrahepatic cholangiocarcinoma on CECT. He underwent a major left hepatectomy. The fourth patient presented with classic symptoms, her imaging suggested bilobar liver metastases from an unknown primary.

Only the fifth patient raised a high index of clinical suspicion for primary hepatic TB. All the patients had no evidence of focus of TB anywhere else in the body thus validating the diagnosis of primary hepatic TB.

The histopathology report in all the cases was suggestive of necrotizing granulomatous inflammation. AFB stain was performed in 5 cases and was negative in all patients. In literature, AFB stain positive only in $40 \%$ of cases. TB - PCR has a sensitivity of $82 \%$ for detecting Mycobacterium tuberculae in tissue samples and should be considered the investigation of choice for confirming the diagnosis in such cases. ${ }^{8,9}$ TB - PCR was not done in our cases since the test is not available at our center. Treatment for primary hepatic TB is in the form of effective ATT, the drugs and regimen to be individualized according to the severity and liver function of the patient. In conclusion, primary hepatic $\mathrm{TB}$ is a rare entity where 
the diagnosis is difficult given confounding clinical features and imaging characteristics. It may mimic primary liver malignancy or metastases in the setting of pre-existing malignancy. This case series stresses the essentiality of having a high index of suspicion and preoperative biopsy in cases where imaging features are equivocal.

\section{REFERENCES}

1. Mert A, Ozaras R, Tabak F, Ozturk R, Bilir M. Localized hepatic tuberculosis. Eur J Intern Med 2003;14:511-512.

2. Park JI. Primary hepatic tuberculosis mimicking intrahepatic cholangiocarcinoma: report of two cases. Ann Surg Treat Res 2015;89:98-101.

3. Levine C. Primary macronodular hepatic tuberculosis: US and CT appearances. Gastrointest Radiol 1990;15:307-309.
4. Brookes MJ, Field M, Dawkins DM, Gearty J, Wilson P. Massive primary hepatic tuberculoma mimicking hepatocellular carcinoma in an immunocompetent host. MedGenMed 2006;8:11.

5. Singh S, Jain P, Aggarwal G, Dhiman P, Singh S, Sen R. Primary hepatic tuberculosis: a rare but fatal clinical entity if undiagnosed. Asian Pac J Trop Med 2012;5:498-499.

6. Kok KY, Yapp SK. Isolated hepatic tuberculosis: report of five cases and review of the literature. J Hepatobiliary Pancreat Surg 1999;6:195-198.

7. Amarapurkar DN, Patel ND, Amarapurkar AD. Hepatobiliary tuberculosis in western India. Indian J Pathol Microbiol 2008;51: 175-181.

8. Singh KK, Muralidhar M, Kumar A, Chattopadhyaya TK, Kapila $\mathrm{K}$, Singh MK, et al. Comparison of in house polymerase chain reaction with conventional techniques for the detection of Mycobacterium tuberculosis DNA in granulomatous lymphadenopathy. J Clin Pathol 2000;53:355-361.

9. Huang WT, Wang CC, Chen WJ, Cheng YF, Eng HL. The nodular form of hepatic tuberculosis: a review with five additional new cases. J Clin Pathol 2003;56:835-839. 